\title{
Introduction to the Design and Appropriation of Knowledge and AI Systems Minitrack
}

\author{
Stefan Smolnik \\ University of Hagen \\ stefan.smolnik@fernuni- \\ hagen.de
}

\author{
Pierre Hadaya \\ Université du Québec à \\ Montréal \\ hadaya.pierre@uqam.ca
}

\author{
W. David Holford \\ Université du Québec à \\ Montréal \\ holford.w david@ \\ $\underline{\text { uqam.ca }}$
}

The objective of this minitrack is to contribute to the body of knowledge that helps scholars and practitioners increase their collective understanding of

(1) how knowledge and artificial intelligence (AI) systems are planned, designed, built, implemented, used, evaluated, supported, upgraded, and evolved;

(2) how knowledge and AI systems impact the context in which they are embedded; and

(3) the human behaviors reflected within and induced through both (1) and (2).

By knowledge and AI systems, we mean systems in which human participants and/or machines perform work (processes and activities) related to the creation, retention, transfer and/or application of knowledge using information, technology, and other resources to produce informational products and/or services for internal or external customers (adapted from Alter 2008). Such systems may include, but are not limited to, knowledge management systems, decision systems, social media, expert systems, machine learning systems, and other AI systems as well as any other ITenabled knowledge processes.

It is the tenth year of the minitrack. We received six papers this year and after a rigorous review process, we accepted three for publication in the proceedings and online presentation at the conference.

The first paper, authored by Camille Grange, examines the societal acceptability of a consequential decision-making system (university admission) to those subject to the decision (i.e., applicants). The author analyzes two key drivers of such systems: the nature of the decision-making agent and the decisionmaking logic used by the agents. Consistent with uniqueness neglect theory, the author proposes that applicants will be more positive toward the use of human agents than to computerized systems. Also, consistent with the theory of procedural justice, the author argues that applicants will find the use of a predetermined logic to be more acceptable than an emerging logic. Eventually, the author presents the details and results of a factorial survey designed to test her theoretical model.

In the second paper, nominated for the HICSS-55 2022 Best Paper Award, Rania Afiouni and Alain Pinsonneault aim to provide a novel and important perspective on job transformation with AI. To do so, the authors build on job crafting theory and extend it to propose a theoretical model explaining the ripple effect of changes from tasks to skills, relationships, and, eventually, job cognition. They introduce the concept of human-in-control (one's perception of his/her ability to deliver desired work outcomes in a work context involving AI) as the goal of the job crafting process. The overall goal is to restore workers' sense of control, that could be disrupted while introducing new AI systems.

The third paper, co-authored by Simon L. Schmidt, Mahei Manhai Li, and Christoph Peters, focuses on hybrid intelligence that can facilitate the solving many IT support issues due to the combination of machine power and individual strengths of humans. The authors derive requirements for an IT support system based on hybrid intelligence (ISSHI), consolidating both literature-based and interview-based requirements with IT-support managers and support agents from different companies into requirements to inform an ISSHI system architecture. This architecture serves as a foundation for future research on hybrid intelligence in IT support.

We wish to thank all of the authors who submitted work for consideration in this minitrack. We also thank the dedicated reviewers for the time and effort they invested in reviewing the papers. We believe that the accepted papers contribute to furthering our understanding on the creation and appropriation of knowledge and AI systems. All author teams will provide presentation slides and/or a presentation video that will be accessible during the conference in January 2022. We hope that all provided materials (papers, slides, videos) inspire discussions, networking, and new ideas and research endeavors. 